\section{Differentiation of Edematous, Tumoral and Normal Areas of Brain Using Diffusion Tensor and Neurite Orientation Dispersion and Density Imaging}

\author{
Masjoodi S. ${ }^{1}$, Hashemi H. ${ }^{2}$, Oghabian M. A. ${ }^{1,3 *}$, Sharifi G. ${ }^{4}$
}

\begin{abstract}
Background: Presurigical planning for glioma tumor resection and radiotherapy treatment require proper delineation of tumoral and peritumoral areas of brain. Diffusion tensor imaging (DTI) is the most common mathematical model applied for diffusion weighted MRI data. Neurite orientation dispersion and density imaging (NODDI) is another mathematical model for DWI data modeling.

Objective: We studied whether extracted parameters of DTI, and NODDI models can be used to differentiate between edematous, tumoral, and normal areas in brain white matter (WM).

Material and Methods: 12 patients with peritumoral edema underwent 3T multi-shell diffusion imaging with b-values of 1000 and 2000 smm-2 in 30 and 64 gradient directions, respectively. We fitted DTI and NODDI to data in manually drawn regions of interest and used their derived parameters to characterize edematous, tumoral and normal brain areas.
\end{abstract}

Results: We found that DTI parameters fractional anisotropy (FA), mean diffusivity (MD), axial diffusivity $(\mathrm{AD})$ and radial diffusivity $(\mathrm{RD})$ all significantly differentiated edematous from contralateral normal brain WM $(\mathrm{p}<0.005)$. However, only FA was found to distinguish between edematous WM fibers and tumor invaded fibers ( $\mathrm{p}$ $=0.001$ ). Among NODDI parameters, the intracellular volume fraction (ficvf) had the best distinguishing power with $(p=0.001)$ compared with the isotropic volume fraction (fiso), the orientation dispersion index (odi), and the concentration parameter of Watson distribution $(\kappa)$, while comparing fibers inside normal, tumoral, and edematous areas.

Conclusion: The combination of two diffusion based methods, i.e. DTI and NODDI parameters can distinguish and characterize WM fibers involved in edematus, tumoral, and normal brain areas with reasonable confidence. Further studies will be required to improve the detectability of WM fibers inside the solid tumor if they hypothetically exist in tumoral parenchyma.

\section{Keywords}

MRI, NODDI, Diffusion Tensor Imaging, Edema, Brain Tumor

\section{Introduction}

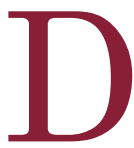
iffusion Magnetic resonance imaging is a unique noninvasive method for in vivo measuring mobility and diffusivity of water molecules [1]. This imaging modality can be used to estimate the micro-structural geometry and the shape of brain white matter indirectly $[2,3]$. Application of Apparent Diffusion Coefficient (ADC), as the most common parameter extracted from Diffusion-Weighted (DW)
${ }^{1}$ Department of Medical Physics and Biomedical Engineering, School of Medicine, Tehran University of Medical Sciences (TUMS), Tehran, Iran

${ }^{2}$ Advanced Diagnostic and Interventional Radi-

ology Research Center

(ADIR), Tehran Univer-

sity of Medical Sciences

(TUMS), Tehran, Iran

${ }^{3}$ Research Center for

Science and Technol-

ogy in Medicine, Imam

Khomeini Hospital Com-

plex, Tehran, Iran

${ }^{4}$ Department of Neu-

rosurgery, Loghman

Hakim Hospital, Shahid

Beheshti University of

Medical Sciences, Teh-

ran, Iran

*Corresponding author: M. A. Oghabian

Biomedical Engineering and Medical Physics group, Faculty of Medicine, Tehran University of Medical Sciences,

Tehran, Iran

E-mail: Oghabian@sina. tums.ac.ir

Received: 5 December 2017 Accepted: 28 March 2018 
images, in different clinical domains such as neuro-oncology and central nervous system (CNS) surgery is increasing [4-6]. It has been shown that there is a negative correlation between ADC quantities and cellularity of tumors [7].

Among many extractable parameters from DWI models, ADC and its relation with two different types of diffusion in tumoral areas, i.e. restricted and hindered diffusion, have been explored in many studies [8]. Indeed, an increase in cellularity causes more restricted diffusion, which is likely to reduce ADC compared to normal WM. On the other hand, the role of edema and necrosis and their partial volume effects should not be neglected $[9,10]$. Hindered diffusion of extracellular water molecules elevates the ADC in tumoral areas so that those regions could not be distinguished from normal apparent white matter.

Fractional anisotropy (FA) is the most known parameter of diffusion tensor imaging (DTI) models and it is used as a stopping criterion in deterministic tractography algorithms for $3 \mathrm{D}$ reconstruction of brain white matter bundles [11]. Application of tractography results for presurgical planning, tumor resection and navigation surgery of the brain is increasing [12-14]. A reduction of FA parameter in peritumoral areas of brain is a challenging problem [15]. To find a proper FA value as the lowest threshold for tractography algorithms, it is necessary to prospect how different tissue types, tumoral, normal apparent ,and peritumoral white matters of brain are separable.

In recent years, the application of neurite orientation dispersion and density imaging (NODDI) model as a multi-compartment model for study of microstructures of brain tissue in the research domain as well as for the clinical use is progressive [16-18]. NODDI model by differentiating intra- and extracellular water molecules's roles in the diffusion process within the nervous fiber boundless and considering an isotropic term for modeling of free water diffusion presents a more proper and sensible model ,and physically interpretable parameters. Multi b-value imaging (multishell-DWI) is a prerequisite condition for applying NODDI model [16].

Tumoral tissues could affect white matted fiber bundles in different ways: displacement, disorientation and disruption and/or invading, and beside each mentioned alterations, edematous areas with significantly reduced FA could be developed around tumors. Some studies have explored the effect of edema on the white matter fiber bundles [19]. One of the oldest classifications for different types of edema was done by Klatzo[20]. According to definition, edema is the result of accumulation of water or related fluids in tissue which leads to increasing the volume of brain parenchyma in that area. The effects of two most famous types of edema, cytotoxic and vasogenic edema, on biochemical and biomechanical characteristics of brain parenchyma have been studied in [21]. Indeed, when encountering with glioma tumors, especially high grade gliomas, damage to Blood Brain Barrier (BBB) increases the water (protein plasma) in extracellular spaces, consequently the diffusion behavior of water molecules will be changed. It is important to differentiate between the white matter fiber tracts in tumoral and edematous areas, in order to plane a safe and efficient surgery; the determination of a border for them is clear because it can affect presurgical plane also radiotherapy treatment planning.

In this study, we will explore differentiability of three different brain tissues, edematous, normal and tumoral areas of brain in glioma patients based on DWI signals applying DTI and NODDI models for the purpose diffusion MRI from 12 patients with glioma tumor acquired, preprocessed and analyzed. Details for data processing and statistical analysis will be explained in the next sections.

\section{Material and Methods}

According to schematic depicted in Figure 1 , after data acquisition and preprocessing of 


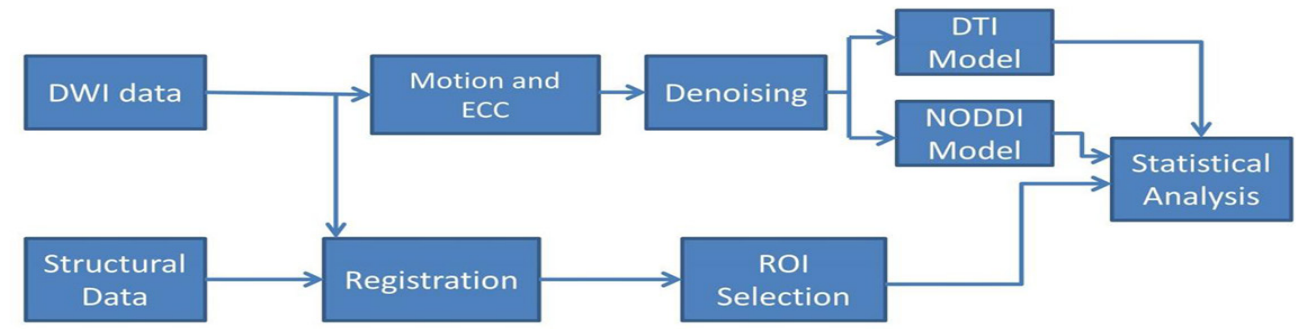

Figure 1: Block diagram of data processing pipeline.

DWI raw data, DTI model was applied on the data. ROI selection was based on conventional MR images, thus before drawing ROIs, structural images were registered on non-diffusion weighted DW image (b0). Next, mean values of parameters FA, MD, AD and RD extracted from DTI model in three regions of interests (ROI) related to edematous, normal and tumoral areas, were calculated. ROIs were drawn within the tumoral area, and special parts of white matter in peritumoral and contralateral normal areas. In the next step, NODDI model was fitted on DWI signals and its corresponding parameters $f_{i c v o}, f_{\text {iso }}$, odi and $\kappa$ in the predetermined ROIs determined. After averaging extracted parameters within all ROIs for each patient, their differentiability, as three different groups, was assessed through proper statistical tests.

\section{Patients}

This retrospective patient study was approved by the institutional review board of Tehran University of Medical Sciences.12 Glioma patients, (average age: $37.8 \pm 13.5$, male/female: 8/4), underwent to a 3 Tesla MRI scanner. All data acquisition was done at Imaging Center of Imam Khomeini complex. Before using patient's data, all images were anonymized, and selected for further diffusion analysis. Further patient details are in Table 1.

\section{Data Acquisition}

MR data acquisition was performed using a 3-T clinical whole-body scanner (TrioTim; Siemens Medical Solutions, Erlangen, Ger-

Table 1: Demographic and clinical characteristics of the patients in this study (GBM: GlioBlastoma Multiform, LGG: Low Grade Glioma, HGG: High Grade Glioma).

\begin{tabular}{cclcl} 
Patient No. & Age (years) & Sex & Diagnosis & Location of Lesion \\
\hline $\mathbf{1}$ & 30 & Male & GBM & Frontal lobe \\
\hline $\mathbf{2}$ & 44 & Female & HGG & Frontal fossa \\
\hline $\mathbf{3}$ & 51 & Female & GBM & Temporo Occipital \\
\hline $\mathbf{4}$ & 32 & Male & GBM & Left Parietal \\
\hline $\mathbf{5}$ & 60 & Male & GBM & Left FrontoTemporal \\
\hline $\mathbf{6}$ & 11 & Female & Astrocytoma & Left Parietal \\
\hline $\mathbf{7}$ & 29 & Male & GBM & Left Temporo Occipital \\
\hline $\mathbf{8}$ & 45 & Male & LGG & Pineal \\
\hline $\mathbf{9}$ & 36 & Male & LGG & Frontal parasagittal \\
\hline $\mathbf{1 0}$ & 23 & Male & LGG & Left frontal cortical \\
\hline $\mathbf{1 1}$ & 43 & Male & HGG & Right temporo frontal \\
\hline $\mathbf{1 2}$ & 50 & Female & HGG & Left parietal
\end{tabular}


many) equipped with the standard 32 channel head coil.

For extracting anatomical details of the brain structure, we applied conventional MR imaging modalities for each patient as follow: a) a three-dimensional (3D) magnetization prepared rapid acquisition gradient echo (MPRAGE) sequence (TR/TE 1800/3.4 ms, $1 \mathrm{~mm}$ isotropic matrix, $256 \times 256 \mathrm{~mm}$ FOV, 176 slices), and b) an axial fluid-attenuated inversion recovery (FLAIR) sequence (TR/TE 8000/104 ms, 3 mm slices).

Diffusion MRI consisted of diffusionweighted echo-planar imaging (EPI) pulse sequences. For our purpose the following parameters were set: TR/TE 9600/101 ms, $128 \times 128$ matrix size, $240 \times 240 \mathrm{~mm}^{2}$ the field of view (FOV), $2 \mathrm{~mm}$ slice thickness, 1502 $\mathrm{Hz} /$ pixel bandwidth. Sixty-eight slices with no intersectional gaps and an isotropic voxel size of $2 \times 2 \times 2 \mathrm{~mm}^{3}$ were acquired. Diffusion gradient encoding vectors were in 30 and 64 directions for $b=1000 \mathrm{smm}^{-2}$, and $b=2000 \mathrm{smm}^{-2}$, respectively. Additionally, two $b=0 \mathrm{smm}^{-2}$ (no diffusion gradient) images were acquired at the start of diffusion encoding gradients. The sequence design was based on balanced diffusion gradients in order to minimize eddy cur- rent artifacts. The patients' heads were fixed in a headrest to minimize artifacts secondary to the unavoidable motion. Figure 2 shows some typical MR images related to subject 1 among patients.

\section{Preprocessing of diffusion MRI im-} ages

After data acquisition, the data sets were preprocessed according to the block diagram shown in Figure 3. Eddy current artifact and patient head motion of DWI data were corrected using the FSL software [22]. The nonlocal-means algorithm [23] was applied for DWI data noise effects reduction and finally DTI and NODDI models were fitted on the corrected data. For working through a unique spatial space all structural data were registered on b0 images.

\section{Data Analysis}

\section{DTI Data Analysis}

Applying Dipy software, the diffusion tensor model was fitted to the data for each voxel of the data using nonlinear least square fitting [24]. After the calculation of principal eigenvalues $\left(\lambda_{1}, \lambda_{2}, \lambda_{3}\right) 4$ maps corresponding to

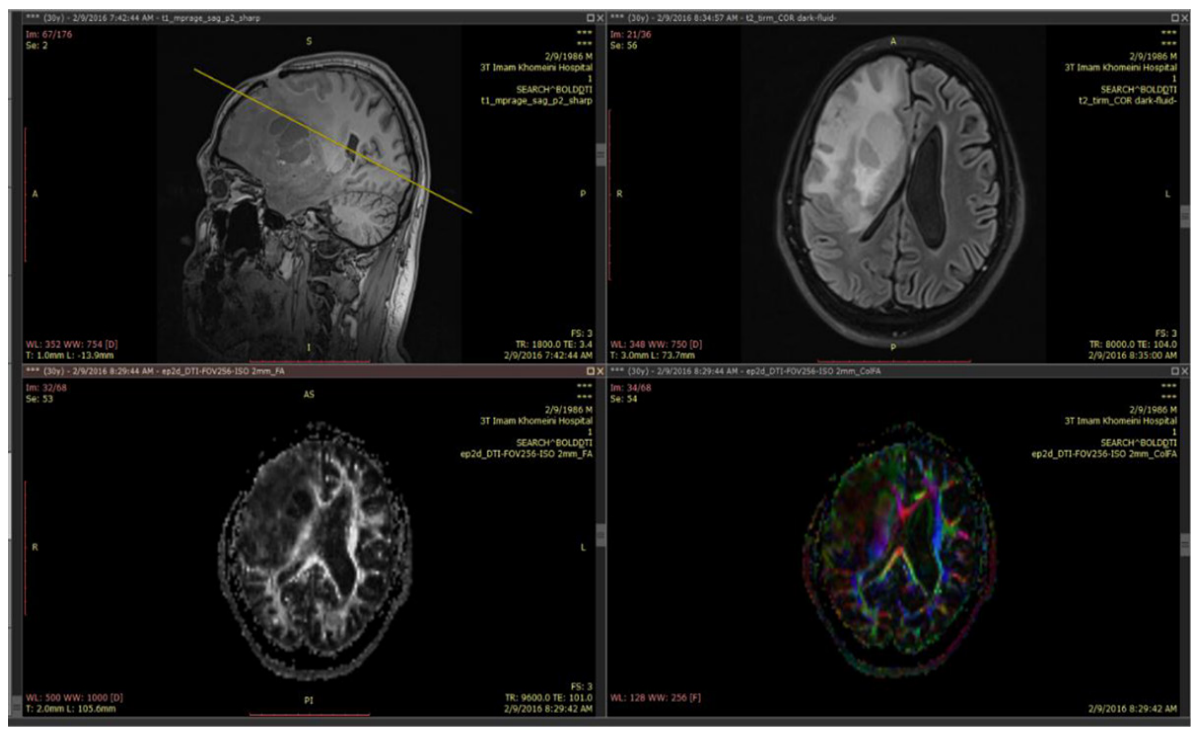

Figure 2: Sample structural T1-weighted (left-above) and Flair (right-above) images in sagital and axial view as well as FA (left-below) and color FA (right below) maps in axial view for subjec1. 


\begin{tabular}{c|c|c|c|c|}
\hline $\begin{array}{c}\text { Data } \\
\text { Acquisition }\end{array}$ & Preprocessing & DW Signal Modeling & ROI & Statistical \\
& (FSI and Dipy) & (Dipy and NODDI & Drawing & Analysis \\
& (ITK-snap) & (SPSS) \\
\hline
\end{tabular}

Figure 3: DWI data analysis pipeline and utilized softwares.

parameters of DTI model, i.e. fractional anisotropy (FA), mean diffusivity (MD), axial diffusivity (AD) and radial diffusivity(RD) were extracted for all patients. FA, MD, AD, and RD maps are related to eigenvalues as follow:

$$
\begin{gathered}
F A=\sqrt{\frac{1}{2} \frac{\left(e_{1}-e_{2}\right)^{2}+\left(e_{2}-e_{3}\right)^{2}+\left(e_{3}-e_{1}\right)^{2}}{\left(e_{1}^{2}+e_{1}^{2}+e_{1}^{2}\right)}} \\
M D=\frac{e_{1}+e_{2}+e_{3}}{3} \\
A D=e_{1} \\
R D=\frac{e_{1}+e_{2}}{2}
\end{gathered}
$$

Figure 4 shows an axial view of the extracted quantitative maps from DTI model for patient \#1.

\section{NODDI Data Analysis}

After two decades from the introduction of diffusion models for living tissues, many different multi-compartments models have been presented, i.e. ball and sticks model, CHARMED model, AxCaliber and many more [25-27]. Among multi-compartment models a model with the name NODDI has been pre- sented and noticed more in recent years [16]. NODDI model assumes that the acquired diffusion signal is composed of three different compartments: intracellular, extracellular and CSF compartments. The mathematical equation for describing NODDI model is as follow: $\boldsymbol{S}=f_{i s o} \boldsymbol{S}_{i s o}+\left(1-f_{i s o}\right)\left(f_{i c} \boldsymbol{S}_{i c}+\left(1-f_{i c}\right) \boldsymbol{S}_{e c}\right)$

in which $f_{i s o}, f_{i c}$ are CSF and the intracellular volume fraction of signals and $S_{i c}, S_{e c}$ and $S_{\text {iso }}$ are intracellular, extracellular and CSF signals, respectively. Figure 5 demonstrates different parameter contrasts estimated from NODDI model for subject \#1. As we can see, the variation of contrasts with respect to DTI contrasts is more obvious.

\section{ROI selection}

Considering anatomical and structural MR images, T1-, T2-weighted and Flair, a radiologist expert with more than 15-years experiences drew three different ROIs for peritumoral, normal and tumoral areas of brain white matters. For this work, applying itk-snap software [28], an ROI was drawn on a part of brain tissue occupied with edema so that it encompassed some parts of white matter tissue of the brain. A typical ROI selection for the dataset related to subject \#1 has been shown in Figure 6. Normal ROI was drawn in a contralateral

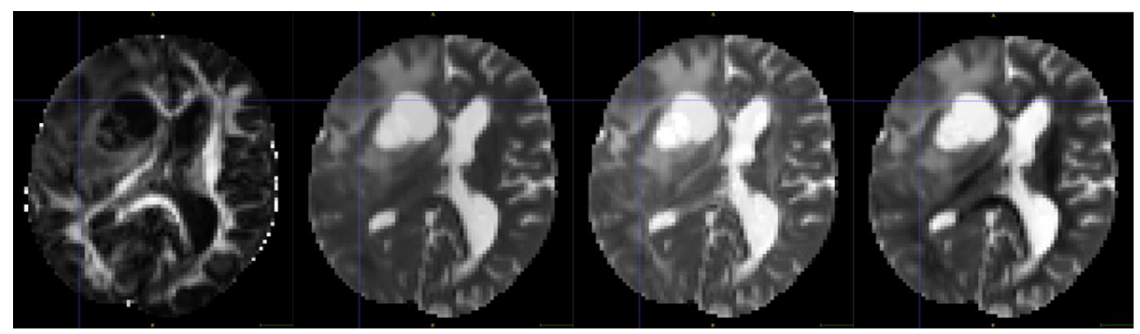

Figure 4: Maps extracted from DTI model. From left to right: FA, MD, AD, and RD. 

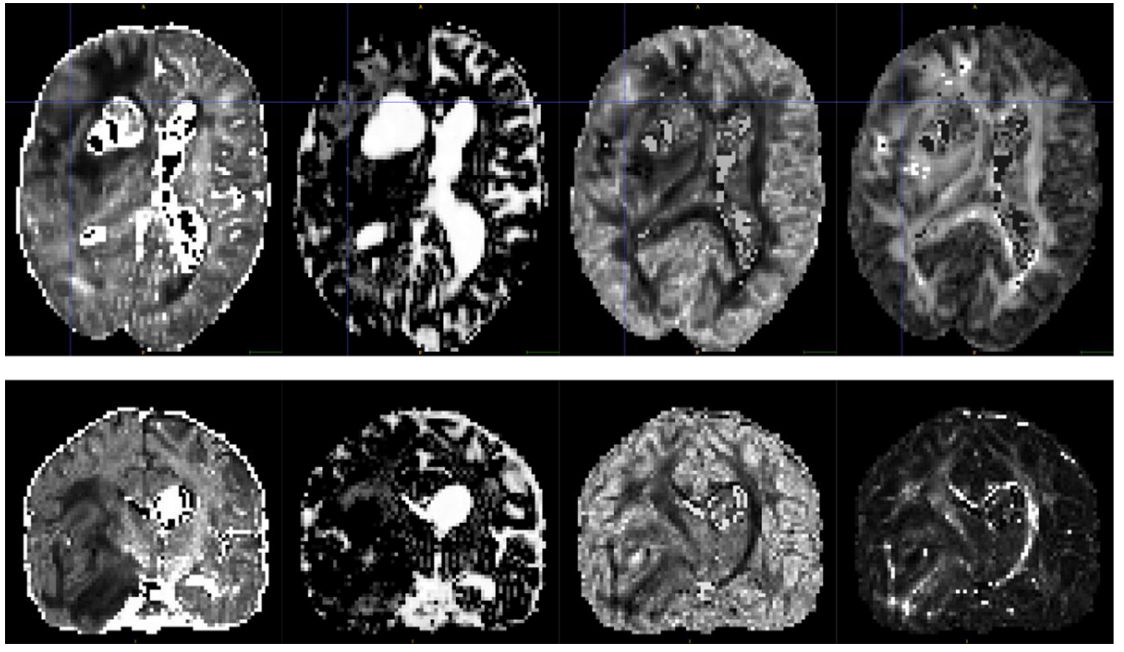

Figure 5: Maps extracted from NODDI model. Above maps from left to right: ficvf, fiso, odi, kappa from a coronal view. Lower maps are like upper row unless they are from a coronal view.

area hemisphere of the brain and it was drawn as same as possible to the edematous area. For tumoral area, the ROI was selected near the core and massive part on the tumor. Because all anatomic images were registered to DWI images, the same ROIs were applied so as to extract the scalar quantities of all DTI an NODDI maps. Finally, after applying ROIs on the maps and getting average, eight different quantities were extracted from each ROI for all patients. Scalar values calculated for each selected ROI were inserted into Table 2 and Table 3 for DTI and NODDI models, respectively.

\section{Statistical Analysis}

We applied nonparametric statistical tests for assessing the differentiability of 3 groups of tissues. On the other hand, comparison should be made among more than two groups. Hence, in the first stage we used Kruskal-Wallis test which corresponds to one-way ANOVAs test [29]. The level of significance was considered to be 0.05 . We applied Kruskal-Wallis on four variables extracted from DTI model and four variables extracted from NODDI model. In the second stage of statistical analysis, we used Mann-Whitney U test as our post-hoc test for a pair test between each group of variables. All statistical analyses were done using SPSS version 24 for windows OS.

Results

In this section, the statistical analysis results

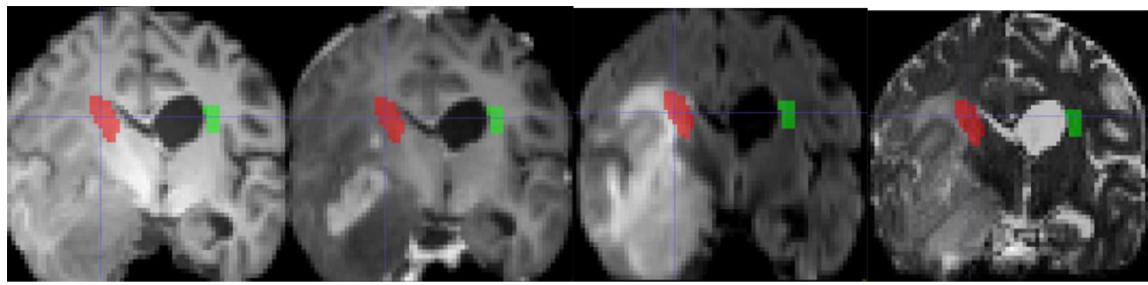

Figure 6: Coronal view of conventional MR images for subject 1. From left to right: T1, T1-post contrast, Flair and T2 with overlaid ROIs. An edematous portion of left corticospinal tract (CST) in red and its normal corresponding on right side in green was drawn as edematous and normal ROIs. 
Table 2: Statistical analysis results for DTI model parameters.

\begin{tabular}{ccccc} 
Kruskal-Wallis Test/ Mann-Whitney Test & FA (0.001) & MD(0.001) & AD(0.001) & RD(0.001) \\
\hline Edematuas vs Normal & 0.005 & 0.001 & 0.001 & 0.001 \\
\hline Edematous vs Tumoral & 0.001 & 0.2 & 0.87 & 0.09 \\
\hline Normal vs Tumoral & 0.001 & 0.001 & 0.007 & 0.001
\end{tabular}

Table 3: non-parametric statistical analysis for NODDI model parameters.

\begin{tabular}{ccccc} 
Kruskal-Wallis Test/ Mann-Whitney Test & $\mathbf{f}_{\text {icvf }}(\mathbf{0 . 0 0 1 )}$ & $\mathbf{f}_{\text {iso }}(\mathbf{0 . 0 2})$ & odi(0.004) & $\mathbf{k a p p a ( 0 . 0 0 8 )}$ \\
\hline Edematoas vs Normal & 0.001 & 0.158 & 0.01 & 0.17 \\
\hline Edematoas vs Tumoral & 0.97 & 0.082 & 0.003 & 0.004 \\
\hline Normal vs Tumoral & 0.001 & 0.014 & 0.22 & 0.45
\end{tabular}

from DTI and NODDI model as explained in the previous sections are represented in Tables 2 and Table 3.

\section{DTI model statistical results}

Here statistical analysis results regarding to DTI model parameters will be described in more details. As we can see from the first row of Table 2, four extracted DTI model variables, FA, MD, $\mathrm{AD}$ and $\mathrm{RD}$ have passed Kruskal-Wallis test, therefore, we can keep on pairwise comparison for our three groups. According to pairwise Mann-Whitney U test, FA can discriminate all groups from each other. FA capability for separating the normal group from the edematous group in comparison to other pairwise comparisons is weaker ( $p$-value $=0.005$ versus $p$-value $=0.001$ ). As it is clear from the third row of the same table, the differentiation of edematous from tumoral group is more challenging. As we can see among four DTI variables only FA and RD can be distinguished between tumoral and edematous areas.

\section{NODDI model statistical results}

The statistical analysis for quantified DWI signals using NODDI model is represented in Table 3. It is clear from first table that unless $f_{\text {iso }}$ parameter that has a relative low sig- nificance with respect to others, the three other variables extracted from NODDI model have passed Kruskal-Wallis statistical test with a high score $(\mathrm{p}<0.01)$. Among other parameters, $f_{i c v f}$ can distinguish the normal class from both other edematous and tumoral classes with p-value $=0.001$. On the other hand, odi and kappa parameters can separate edematous and tumoral groups from each other with $\mathrm{p}=0.003$ and $\mathrm{p}=0.004$ respectively, and finally $f_{\text {iso }}$ map is a good contrast for separating tumoral from the normal region.

\section{Discussion}

The effects of tumor on brain white matter are not distinguishable using conventional MRI (T1, T2, Flair), also those images do not provide any information about directionality and orientations of fiber bundles. Presurgical functional MRI and intraoperative electrical stimulations could help to the identification of eloquent regions of gray matter,but they do not give any information about white matter. A more complete presurgical planning requires some data about the white matter fiber bundles that surround the lesion. Awareness about the spatial relation between tumoral area and fiber tracts can help neurosurgeon to retain the functional relations of the brain more precisely.

An outburst and developing of tumoral cells 
by altering the micro structural organization of the brain that lead to tissue alteration change the anatomy of the brain. One of these physiologic changes around the tumoral environment is a change of water content in parenchyma of the brain variation of velocity and the direction of water molecules diffusion. From the other hand, conventional MRI underestimates the tumoral area, thus applying more advanced imaging methods is necessary. Applications of diffusion MRI for clinical goals are one of those more advanced strategies. There are many studies to assess brain tumors using diffusion MRI.

In this study, using a 3T scanner we acquired a two shell diffusion dataset from 12 patients with brain tumors to explore if we can distinguish among tumoral, normal and edematous areas within white matter or not. Twoshell data set involves 30 and 64 volumes of brain corresponding to b-values of 1000 and 2000, respectively. For modeling the acquired data, we used the well-known and most common DTI model and a more advanced model known as NODDI.

All the parameters extracted from DTI mod$\mathrm{el}$, in the edematous ROIs of white matters were significantly different from corresponding areas in the normal areas within the other hemisphere. The reduction of FA in the edematous and tumoral areas is due to activities of tumoral cells that affect the geometrical microstructure of the tissues within and around them. Indeed, with increasing the amount of water and changing the size of intracellular and extracellular spaces around the fiber bundles could alter the diffusivity constants in all directions. A comparison between $\mathrm{AD}$ and RD quantities in the edematous areas with corresponding values in the normal regions shows that an increase in the diffusivity in both directions (axial and perpendicular to the axons) is explicit but for RD this growth is about two times more than healthy parts. These findings are compatible with the reports presented by [30].
The estimated mathematical relationship among FA, MD, AD, and RD satisfies the statistical analysis results. The large difference between the growth rate of $\mathrm{AD}$ and $\mathrm{RD}$ in edematous areas with respect to healthy parts of white matter refers to this note that the diffusion alterations are not isotropic, the point that has been expressed clearly in a paper titled "Is the swelling in brain edema isotropic or anisotropic?" [31]

In comparison to DTI model, extracted maps from NODDI model from the variability and information enrichment point of view are more exhaustive. This variability of NODDI model for distinguishing among edematous, normal and tumoral groups is originated from the intrinsic difference between two models. NODDI model with a more complicated mathematical model could prepare some output parameters with fewer redundancies. On the other hand, there is not a closed form mathematical relationship among different parameters extracted from NODDI model.

Among all eight parameters extracted from DTI and NODDI models, only 2 parameters could distinguish between edematous and normal as well as edematous and tumoral areas, FA extracted from DTI and ODI from NODDI. Proliferation of tumoral cells within and around fiber tracts reduces their parallelism, convergence and regulation and this factor is the main cause for odi alteration. The reduction of extracellular space is another factor that affects odi values. MD could separate the normal group from two other ones but could not distinguish between edematous and tumoral areas; it means that water volume and its diffusivity factors have increased in those two areas.

As we know diffusion weighted imaging is capable to detect micro structural characteristics of brain neuronal tissues. Indeed, because the semi-cylindrical shape of white matter fibers, diffusion of water is not isotropic for white matter bundles, therefore, within the high cellularity environments we expect to see 
higher FAs and lower MDs with respect to areas with lower cellular densities and concentrations. One limitation of this study is that we did not consider distinguishing tumoral areas in details, the areas with proliferating, necrosis and affected by invasion from one hand and the separation of edematous from infiltrative regions from the other hand are some problems that could be explored in future works. Applying classification methods for clustering and segmenting different parts of glioma is in our next future list of works. Another model that we can work on using our multi-shell data is diffusion kurtosis imaging (DKI) [32].

\section{Conclusion}

In this work, we evaluated two different models to separate normal apparent, edematous and tumoral white matters of brain. Each model has its specific limitations and advantages. One of most interested applications of DTI model is tractography of fiber tracts for the $3 \mathrm{D}$ visualization of fiber bundles paths. Application of other quantized parameters like odi extracted from NODDI model instead of FA map as a stopping parameter in the tractography algorithms could be explored in the future works.

\section{Acknowledgment}

This study is part of a $\mathrm{PhD}$ thesis supported by Tehran University of Medical sciences (grant No: 93-03-30-26078).

\section{Conflict of Interest}

\section{None}

\section{References}

1. Le Bihan D, Breton E, Lallemand D, Grenier $P$, Cabanis E, Laval-Jeantet M. MR imaging of intravoxel incoherent motions: application to diffusion and perfusion in neurologic disorders. Radiology. 1986;161:401-7. doi: 10.1148/radiology.161.2.3763909. PubMed PMID: 3763909.

2. Lenglet $C$, Deriche $R$, Faugeras 0 , editors. Inferring white matter geometry from diffusion tensor MRI: Application to connectivity mapping. European Conference on Computer Vision; 2004: Springer.
P.127-140.

3. Ramirez-Manzanares A, Rivera M, Vemuri BC, Carney $P$, Mareci T. Diffusion basis functions decomposition for estimating white matter intravoxel fiber geometry. IEEE Trans Med Imaging. 2007;26:1091102. doi: 10.1109/TMI.2007.900461. PubMed PMID: 17695129.

4. Kono K, Inoue $\mathrm{Y}$, Nakayama $\mathrm{K}$, Shakudo M, Morino $\mathrm{M}$, Ohata $\mathrm{K}$, et al. The role of diffusion-weighted imaging in patients with brain tumors. AJNR Am J Neuroradiol. 2001;22:1081-8. PubMed PMID: 11415902.

5. Sundgren $P C$, Dong $Q$, Gomez-Hassan $D$, Mukherji SK, Maly P, Welsh R. Diffusion tensor imaging of the brain: review of clinical applications. Neuroradiology. 2004;46:339-50. doi: 10.1007/s00234003-1114-x. PubMed PMID: 15103435.

6. Sundgren PC, Fan X, Weybright $P$, Welsh RC, Carlos RC, Petrou M, et al. Differentiation of recurrent brain tumor versus radiation injury using diffusion tensor imaging in patients with new contrast-enhancing lesions. Magn Reson Imaging. 2006;24:1131-42. doi: 10.1016/j.mri.2006.07.008. PubMed PMID: 17071335.

7. Chenevert TL, Sundgren PC, Ross BD. Diffusion imaging: insight to cell status and cytoarchitecture. Neuroimaging Clin N Am. 2006;16:619-32. doi: 10.1016/j.nic.2006.06.005. PubMed PMID: 17148023.

8. White NS, McDonald CR, Farid N, Kuperman JM, Kesari S, Dale AM. Improved conspicuity and delineation of high-grade primary and metastatic brain tumors using "restriction spectrum imaging": quantitative comparison with high B-value DWI and ADC. AJNR Am J Neuroradiol. 2013;34:95864, S1. doi: 10.3174/ajnr.A3327. PubMed PMID: 23139079; PubMed Central PMCID: PMC4146398.

9. Sugahara T, Korogi Y, Kochi M, Ikushima I, Shigematu $Y$, Hirai T, et al. Usefulness of diffusionweighted MRI with echo-planar technique in the evaluation of cellularity in gliomas. J Magn Reson Imaging. 1999;9:53-60. PubMed PMID: 10030650.

10. Maier SE, Sun Y, Mulkern RV. Diffusion imaging of brain tumors. NMR Biomed. 2010;23:849-64. doi: 10.1002/nbm.1544. PubMed PMID: 20886568; PubMed Central PMCID: PMC3000221.

11. Basser PJ, Pajevic S, Pierpaoli C, Duda J, Aldroubi A. In vivo fiber tractography using DT-MRI data. Magn Reson Med. 2000;44:625-32. PubMed PMID: 11025519.

12. Pujol S, Wells W, Pierpaoli C, Brun C, Gee J, Cheng G, et al. The DTI Challenge: Toward Standardized Evaluation of Diffusion Tensor Imaging Tractography for Neurosurgery. J Neuroimaging. 2015;25:875-82. doi: 10.1111/jon.12283. PubMed PMID: 26259925; PubMed Central PM- 
CID: PMC4641305.

13. Stadlbauer A, Nimsky C, Buslei R, Salomonowitz $\mathrm{E}$, Hammen T, Buchfelder M, et al. Diffusion tensor imaging and optimized fiber tracking in glioma patients: Histopathologic evaluation of tumor-invaded white matter structures. Neuroimage. 2007;34:949-56. doi: 10.1016/j.neuroimage.2006.08.051. PubMed PMID: 17166744.

14. Dimou S, Battisti RA, Hermens DF, Lagopoulos J. A systematic review of functional magnetic resonance imaging and diffusion tensor imaging modalities used in presurgical planning of brain tumour resection. Neurosurg Rev. 2013;36:205-14. doi: 10.1007/s10143-012-0436-8. PubMed PMID: 23187966.

15. Chen Z, Tie Y, Olubiyi O, Zhang F, Mehrtash A, Rigolo $\mathrm{L}$, et al. Corticospinal tract modeling for neurosurgical planning by tracking through regions of peritumoral edema and crossing fibers using twotensor unscented Kalman filter tractography. Int J Comput Assist Radiol Surg. 2016;11:1475-86. doi: 10.1007/s11548-015-1344-5. PubMed PMID: 26762104; PubMed Central PMCID: PMC4942409.

16. Zhang H, Schneider T, Wheeler-Kingshott CA, Alexander DC. NODDI: practical in vivo neurite orientation dispersion and density imaging of the human brain. Neuroimage. 2012;61:1000-16. doi: 10.1016/j.neuroimage.2012.03.072. PubMed PMID: 22484410.

17. Colgan N, Siow B, O'Callaghan JM, Harrison IF, Wells JA, Holmes HE, et al. Application of neurite orientation dispersion and density imaging (NODDI) to a tau pathology model of Alzheimer's disease. Neuroimage. 2016;125:739-44. doi: 10.1016/j.neuroimage.2015.10.043. PubMed PMID: 26505297; PubMed Central PMCID: PMC4692518.

18. Kamagata K, Hatano T, Aoki S. What is NODDI and what is its role in Parkinson's assessment? United Kingdom: Taylor \& Francis; 2016.

19. Jellison BJ, Field AS, Medow J, Lazar M, Salamat MS, Alexander AL. Diffusion tensor imaging of cerebral white matter: a pictorial review of physics, fiber tract anatomy, and tumor imaging patterns. AJNR Am J Neuroradiol. 2004;25:356-69. PubMed PMID: 15037456.

20. Klatzo I. Presidental address. Neuropathological aspects of brain edema. J Neuropathol Exp Neurol. 1967;26:1-14. PubMed PMID: 5336776.

21. Kuroiwa T, Ueki $M$, Chen $Q$, Suemasu $H$, Taniguchi I, Okeda R. Biomechanical characteristics of brain edema: the difference between vasogenic-type and cytotoxic-type edema. Acta Neurochir Suppl (Wien). 1994;60:158-61. PubMed PMID: 7976533.

22. Jenkinson M, Beckmann CF, Behrens TE, Woolrich MW, Smith SM. FsI. Neuroimage. 2012;62:782-90. doi: 10.1016/j.neuroimage.2011.09.015. PubMed
PMID: 21979382.

23. Coupé $P$, Yger $P$, Prima $S$, Hellier $P$, Kervrann $C$, Barillot $C$. An optimized blockwise nonlocal means denoising filter for 3-D magnetic resonance images. IEEE Trans Med Imaging. 2008;27:425-41. doi: 10.1109/tmi.2007.906087.

24. Garyfallidis E, Brett M, Amirbekian B, Rokem A, van der Walt S, Descoteaux M, et al. Dipy, a library for the analysis of diffusion MRI data. Front Neuroinform. 2014;8:8. doi: 10.3389/fninf.2014.00008. PubMed PMID: 24600385; PubMed Central PMCID: PMC3931231.

25. Pierpaoli C, Jones D, editors. Removing CSF contamination in brain DT-MRIs by using a two-compartment tensor model. Proc International Society for Magnetic Resonance in Medicine 12th Scientific meeting ISMRM04; 2004.

26. Assaf $Y$, Basser PJ. Composite hindered and restricted model of diffusion (CHARMED) MR imaging of the human brain. Neuroimage. 2005;27:4858. doi: 10.1016/j.neuroimage.2005.03.042. PubMed PMID: 15979342.

27. Assaf $Y$, Blumenfeld-Katzir T, Yovel Y, Basser PJ. AxCaliber: a method for measuring axon diameter distribution from diffusion MRI. Magn Reson Med. 2008;59:1347-54. doi: 10.1002/mrm.21577. PubMed PMID: 18506799; PubMed Central PMCID: PMC4667732.

28. Yushkevich PA, Piven J, Hazlett HC, Smith RG, Ho $\mathrm{S}$, Gee JC, et al. User-guided 3D active contour segmentation of anatomical structures: significantly improved efficiency and reliability. Neuroimage. 2006;31:1116-28. doi: 10.1016/j.neuroimage.2006.01.015. PubMed PMID: 16545965.

29. Kruskal WH, Wallis WA. Use of ranks in one-criterion variance analysis. Journal of the American statistical Association. 1952;47:583-621. doi: 10.1 080/01621459.1952.10483441.

30. Min Z-g, Niu C, Rana N, Ji H-m, Zhang M. Differentiation of pure vasogenic edema and tumorinfiltrated edema in patients with peritumoral edema by analyzing the relationship of axial and radial diffusivities on 3.0 T MRI. Clin Neurol Neurosurg. 2013;115:1366-70. doi: 10.1016/j.clineuro.2012.12.031.

31. Kuroiwa $T$, Ueki $M$, Chen $Q$, Ichinose $S$, Okeda R. Is the swelling in brain edema isotropic or anisotropic? Acta Neurochir Suppl (Wien). 1994;60:155-7. PubMed PMID: 7976532.

32. Jensen JH, Helpern JA, Ramani A, Lu H, Kaczynski K. Diffusional kurtosis imaging: the quantification of non-gaussian water diffusion by means of magnetic resonance imaging. Magn Reson Med. 2005;53:1432-40. doi: 10.1002/mrm.20508. PubMed PMID: 15906300. 